\title{
A NOTE ON COMMON FIXED POINTS BY ALTERING DISTANCES
}

\author{
R. P. PANT, K. JHA AND A. B. LOHANI
}

\begin{abstract}
The purpose of this paper is to provide answer to an open problem due to Sastry et al. [1].
\end{abstract}

\section{Introduction}

Recently, Sastry et al. [1] obtained conditions for the existence of unique common fixed point for weakly commuting pairs of self mappings in a complete metric space, by altering distances between points. Also, Sastry proved a common fixed point theorem, introducing the notion of certain control function in order to alter distance between the points. Sastry et al. [1] established a unique common fixed point theorem for four self mappings by applying the following notions:

Definition 1.1. A control function $\psi$ is defined as $\psi: \Re^{+} \rightarrow \Re^{+}$which is continuous at zero, monotonically increasing, $\psi(2 t) \leq 2 \psi(t)$ and $\psi(t)=0$ if, and only if $t=0$. It is noted that this function $\psi$ need not be sub-additive.

Definition 1.2. Two self mappings $A$ and $S$ of a metric space $(X, d)$ are called weakly commuting if $d(A S x, S A x) \leq d(A x, S x)$ for each $x$ in $X$. This condition implies that $A S x=S A x$ whenever $A x=S x$.

Definition 1.3. Two self mappings $A$ and $S$ of a metric space $(X, d)$ are called $\psi$-compatible if $\operatorname{Lim}_{n} \psi\left(d\left(A S x_{n}, S A x_{n}\right)\right)=0$ whenever $\left\{x_{n}\right\}$ is a sequence such that $\operatorname{Lim}_{n} A x_{n}=\operatorname{Lim}_{n} S x_{n}=t$ for some $t$ in $X$.

Sastry et al. [1] proved the following theorem:

Theorem (2.4. of [1]) Let $(A, S)$ and $(B, T)$ be weakly commuting pairs of self mappings of a complete metric space $(X, d)$ and function $\psi$ be as in definition (1.1) satisfying

(i) $A X \subset T X, B X \subset S X$ and

(ii) there exists $h$ in $[0,1)$ such that $\psi(d(A x, B y)) \leq h M_{\psi}(x, y)$ for all $x, y$ in $X$.

Received January 4, 2002.

2000 Mathematics Subject Classification. 47H10.

Key words and phrases. Complete metric space, Cauchy sequence, compatible maps, weakly commuting maps and common fixed points. 
Suppose that $A$ and $S$ are $\psi$-compatible and $S$ is continuous. Then $A, B, S$ and $T$ have a unique common fixed point.

This theorem is valid if we assume $B$ and $T$ are $\psi$-compatible and $T$ is continuous, instead of similar restrictions on $A$ and $S$.

On the basis of theorem 2.4 of [1], Sastry posed the following open problem:

Is theorem 2.4 of [1] valid if we replace continuity of $S$ by continuity of $A$ or continuity of $T$ by continuity of $B$ ?

In the present paper, we prove a common fixed point theorem which provides an affirmative answer to the above question on the existence of fixed point. Also, we state the following lemma (2.3 of [1]) which is used in the main theorem.

Lemma 1.4. Let $f: \Re^{+} \rightarrow \Re^{+}$be increasing, continuous at the origin and vanishing only at zero. Then $\left\{t_{n}\right\} \subset \Re^{+}$and $f\left(t_{n}\right) \rightarrow 0$ implies that $t_{n} \rightarrow 0$.

\section{Main Theorem}

Theorem 2.1. Let $(A, S)$ and $(B, T)$ be weakly commuting pairs of self mappings of a complete metric space $(X, d)$ and function $\psi$ be as in definition (1.1) satisfying

(i) $A X \subset T X, B X \subset S X$ and

(ii) there exists $h$ in $[0,1)$ such that $\psi(d(A x, B y)) \leq h M_{\psi}(x, y)$ for all $x, y$ in $X$.

Suppose that $A$ and $S$ are $\psi$-compatible and $A$ is continuous. Then $A, B, S$ and $T$ have a unique common fixed point.

Proof. Let $x_{0}$ be any fixed point in $X$. Define sequences $\left\{x_{n}\right\}$ and $\left\{y_{n}\right\}$ in $X$ given by the rule

$$
y_{2 n}=A x_{2 n}=T x_{2 n+1} \quad \text { and } \quad y_{2 n+1}=B x_{2 n+1}=S x_{2 n+2} .
$$

This can be done by virtue of (i). Then applying the same proof as that in the theorem 2.1 of Sastry et al. [1], we can show that $\left\{y_{n}\right\}$ is a Cauchy sequence. Since $X$ is a complete metric space, there is a point $z$ in $X$ such that

$$
y_{2 n}=A x_{2 n}=T x_{2 n+1} \rightarrow z \quad \text { and } \quad y_{2 n+1}=B x_{2 n+1}=S x_{2 n+1} \rightarrow z .
$$

Now, suppose that $(A, S)$ is $\psi$-compatible then we have

$$
A x_{2 n} \rightarrow z \text { and } S x_{2 n} \rightarrow z \text { implies that } \operatorname{Lim}_{n} \psi\left(d\left(A S x_{2 n}, S A x_{2 n}\right)\right)=0 .
$$

Also, since $A$ is continuous, so by (2.1.2), we get

$$
A A x_{2 n} \rightarrow A z \quad \text { and } \quad A S x_{2 n} \rightarrow A z \quad \text { as } \quad n \rightarrow \infty .
$$

We claim that $\operatorname{Lim}_{n} S A x_{2 n}=A z$. Using (2.1.3), we get $\psi\left(d\left(S A x_{2 n}, A z\right)\right) \leq \psi\left(d\left(S A x_{2 n}\right.\right.$, $\left.\left.A S x_{2 n}\right)+d\left(A S x_{2 n}, A z\right)\right) \rightarrow 0$ as $n \rightarrow \infty$. Thus, using the above lemma (1.4), $d\left(S A x_{2 n}, A z\right)$ 
$\rightarrow 0$ as $n \rightarrow \infty$, and so $\operatorname{Lim}_{n} S A x_{2 n}=A z$. Also, since $A X \subset T X$, for each $n$, there exists $w_{2 n}$ in $X$ such that $A A x_{2 n}=T w_{2 n}$ and $A A x_{2 n}=T w_{2 n} \rightarrow A z$. Thus, $A A x_{2 n} \rightarrow$ $A z, S A x_{2 n} \rightarrow A z, A S x_{2 n} \rightarrow A z$ and $T w_{2 n} \rightarrow A z$ as $n \rightarrow \infty$. Again, we claim that $\operatorname{Lim}_{n} B w_{2 n} \rightarrow A z$. If not, then there exists $\varepsilon>0$ and a subsequence $\left\{n_{k}\right\}$ such that $d\left(A A x_{2 n_{k}}, B w_{2 n_{k}}\right)>\varepsilon$ and $\psi\left(d\left(S A x_{2 n_{k}}, A S x_{2 n_{k}}\right)\right)<\varepsilon$ for all $n_{k}$. Therefore,

$$
\begin{aligned}
\psi(\varepsilon) & \leq \psi\left(d\left(A A x_{2 n_{k}}, B w_{2 n_{k}}\right)\right) \\
& \leq h M_{\psi}\left(A x_{2 n_{k}}, w_{2 n_{k}}\right) \\
& =h \max \left\{\psi\left(d\left(S A x_{2 n_{k}}, T w_{2 n_{k}}\right)\right), \psi\left(d\left(A A x_{2 n_{k}}, S A x_{2 n_{k}}\right)\right), \psi\left(d\left(B w_{2 n_{k}}, T w_{2 n_{k}}\right)\right),\right. \\
& \left.\quad\left[\psi\left(d\left(A A x_{2 n_{k}}, T w_{2 n_{k}}\right)\right)+\psi\left(d\left(B w_{2 n_{k}}, S A x_{2 n_{k}}\right)\right)\right] / 2\right\} \\
& =h \max \left\{\psi\left(d\left(B w_{2 n_{k}}, T w_{2 n_{k}}\right)\right),\left[\psi\left(d\left(B w_{2 n_{k}}, S A x_{2 n_{k}}\right)\right)\right] / 2\right\}, \\
& =h \psi\left(d\left(B w_{2 n_{k}}, A A x_{2 n_{k}}\right)\right), \\
& <\psi\left(d\left(B w_{2 n_{k}}, A A x_{2 n_{k}}\right)\right), \quad \text { a contradiction. Hence } \operatorname{Lim}_{n} B w_{2 n}=A z .
\end{aligned}
$$

We claim that $A z=S z$. For this, using (ii), we get

$$
\begin{aligned}
\psi\left(d\left(S z, B w_{2 n}\right)\right) & \leq h M_{\psi}\left(z, w_{2 n}\right) \\
& =h \max \left\{\psi\left(d\left(S z, T w_{2 n}\right)\right), \psi(d(A z, S z)), \psi\left(d\left(B w_{2 n}, T w_{2 n}\right)\right),\right. \\
& {\left.\left[\psi\left(d\left(A z, T w_{2 n}\right)\right)+\psi\left(d\left(B z, S w_{2 n}\right)\right)\right] / 2\right\}, } \\
& =h \max \left\{\psi\left(d\left(S z, T w_{2 n}\right)\right), \psi(d(A z, S z)),\left[\psi\left(d\left(S z, B w_{2 n}\right)\right)\right] / 2\right\} .
\end{aligned}
$$

Letting $n \rightarrow \infty$, we get $\psi(d(S z, A z)) \leq h \max \{\psi(d(S z, A z)), \quad[\psi(d(S z, A z))] / 2\}=$ $h \psi(d(S z, A z))$, a contradiction.

Thus we have

$$
A z=S z \text {. }
$$

Since $A X \subset T X$, there exists some $w$ in $X$ such that $A z=T w$. Therefore, we have

$$
A z=S z=T w .
$$

Moreover, we show that $A z=B w$. Suppose on the contrary that $A z \neq B w$. Then, using (ii), we get

$\psi(d(A z, B w))$

$\leq h M_{\psi}(z, w)$

$=h \max \{\psi(d(S z, T w)), \psi(d(A z, S z)), \psi(d(B w, T w)),[\psi(d(A z, T w))+\psi(d(B z, S w))] / 2\}$,

$=h \max \{\psi(d(B w, A z)),[\psi(d(B w, A z))] / 2\}$,

$=h \psi(d(B w, A z))$, a contradiction.

Therefore,

$$
A z=B w \text {. Hence } \quad A z=S z=T w=B w .
$$

Since $A$ and $S$ are weakly commuting, we have by (2.1.7), $A S z=S A z$ and hence

$$
A A z=A S z=S A z=S S z
$$


and by the weakly commuting property of $B$ and $T$, we get

$$
B B w=B T w=T B w=T T w .
$$

We now show that $A A z=A z$. Suppose that $A A z \neq A z$ then by (ii), we get

$$
\psi(d(A z, A A z))=\psi(d(B w, A A z)) \leq h M_{\psi}(A z, w)=h \psi(d(A z, A A z)),
$$

(using (2.1.7) and (2.1.8)), a contradiction. Hence $A A z=A z$. Also, we have $A A z=S A z$. Therefore, $A z$ is a common fixed point of $A$ and $S$. Again, suppose that $B B w \neq B w$. Then using (ii), we get

$$
\begin{aligned}
\psi(d(B w, B B w)) & =\psi(d(A z, B B w)) & & (\text { by }(2.1 .6)) \\
& \leq h M_{\psi}(z, B w) & & \\
& =h \psi(d(B w, B B w)), & & \text { (by using }(2.1 .7) \text { and }(2.1 .9)) \\
& <\psi(d(B w, B B w)), & & \text { a contradiction. }
\end{aligned}
$$

Hence $B B w=B w$ and since $T B w=B B w$, we have $B w$ being a common fixed point for $B$ and $T$. Finally, since $A z=B w$, we have $A z$ as a common fixed point for $A, B, S$ and $T$. Moreover, the uniqueness of a common fixed point follows from (ii).

This completes the proof of the theorem.

The proof is similar when the pair $(B, T)$ is assumed $\psi$-compatible and $B$ is continuous.

\section{References}

[1] K. P. R. Sastry, S. V. R. Naidu, G. V. R. Babu and G. A. Naidu; Generalization of common fixed point theorems for weakly commuting mappings by altering distances; Tamkang Journal of Mathematics 31(2000), 243-250.

Department of Mathematics, Kumaon University, D. S. B. Campus, Nainital-263002, Uttaranchal, India.

Permanent Affiliation: Department of Mathematical Sciences, Kathmandu University, P. O. Box 6250, Kathmandu, Nepal.

Department of Mathematics, Kumaon University, D. S. B. Campus, Nainital-263002, Uttaranchal, India. 